Copyright (C) 2013 IEEE. Personal use of this material is permitted. Permission from IEEE must be obtained for all other uses, in any current or future media, including reprinting/republishing this material for advertising or promotional purposes, creating new collective works, for resale or redistribution to servers or lists, or reuse of any copyrighted component of this work in other works. 


\title{
On the use of inversion formulae for the synthesis of discrete PID controllers
}

\author{
Stefania Cuoghi and Lorenzo Ntogramatzidis
}

\begin{abstract}
This paper presents a new set of formulae for the design of discrete proportional-integral-derivative (PID) controllers under requirements on steady-state performance and robustness specifications, such as the phase and the gain margins, as well as the gain crossover frequency. The proposed technique has the advantage of avoiding trial-anderror procedures or approximations connected to an a posteriori discretisation. This method can also be implemented as a graphical design procedure in the Nyquist plane.
\end{abstract}

\section{INTRODUCTION}

It is a commonly accepted fact that PID controllers are by far the most utilised compensators in control enginnering. Indeed, according to recent and independent estimates, of all compensators employed in industrial process control, those belonging to the family of PID controllers are used in 95$97 \%$ of all cases [1]-[2].

Over the years, countless tuning techniques have been proposed for PID controllers, which differ in complexity, flexibility, and amount of knowledge required on the mathematical model of the process to be controlled. Many surveys and textbooks have been entirely devoted to these techniques, see e.g. [5]-[8] and the references therein.

In recent times, a new stream of literature flourished on the design techniques for PID controllers under frequency domain specifications, see [3], [9]-[13], [20]-[21], and the references cited therein. In particular, much effort has been devoted to the computation of the parameters of the PID controllers that guarantee desired values of the gain/phase margins and of the crossover frequency.

An important design method that has been introduced for the design of first and second order compensators is based on simple closed-form formulae, often referred to as inversion formulae, which explicitly express the parameters of the controller in terms of the design specifications given by the phase/gain margins and the corresponding crossover frequencies. This approach was first presented for lead, lag and lead-lag (notch) networks, [16], [17], see also [18], [19]. In [15] it was shown that simple inversion formulae could be established for continuous-time PID controllers for the computation of their parameters that exactly meet specifications on the steady-state performance, stability margins

Partially supported by the Australian Research Council under the grant FT120100604.

S. Cuoghi is with the Technical School E. Fermi, Modena, Italy. E-mails: stefania.cuoghi@unimore.it

L. Ntogramatzidis is with the Department of Department of Mathematics and Statistics, Curtin University, Perth, WA, Australia. E-mail: L.Ntogramatzidisecurtin.edu.au.

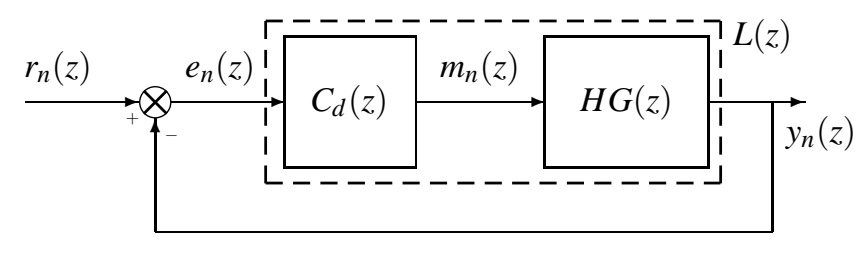

Fig. 1. The considered block scheme for the discrete-time case.

and crossover frequencies, without the need to resort to approximations for the transfer function of the plant.

The goals of this paper are twofold. First, we introduce and investigate a set inversion formulae for discrete PID controllers, motivated by the fact that in industrial applications discrete time is usually preferable to continuous time, being more directly connected to implementation. The second aim is to investigate the graphical counterpart of this method, by showing that the calculation of the parameters of the controller can be carried out with a simple and insightful geometric construction on Nyquist plots, and therefore appears to be very suitable for educational purposes.

The use of inversion formulae presents several advantages, compared with traditional design techniques. First, unlike other analytical synthesis methods [26], steady-state performance specifications can be handled easily. Moreover, the desired phase/gain margins and crossover frequency can be achieved exactly, without the need for trial-and-error or approximations of the plant dynamics. Furthermore, the explicit formulae presented here can be exploited for the self-tuning of the controller. Notice also that the inversion formulae presented here are straightforwardly implementable as MATLAB ${ }^{\circledR}$ routines.

Importantly, the inversion formulae that deliver the parameters of the PID controller as a function of the specifications only depend on the magnitude and argument of the frequency response of the system to be controlled at the desired crossover frequency. As such, this method can be used in conjunction with a graphical method based on any of the standard diagrams for the representation of the dynamics of the frequency response, e.g., the Bode, Nyquist or Nichols diagrams.

\section{DisCRETE PID CONTROLLERS}

Referring to the block scheme of Fig. 1, $H G(z)$ represents the transfer function of the discrete system to be controlled, which is given by the $z$-transform of a zero-order hold $H_{0}(s)$ and a continuous-time plant $G(s)$. Thus, we can write

$$
H G(z)=\mathscr{Z}\left[H_{0}(s) G(s)\right],
$$


where $\mathscr{Z}[\cdot]$ represents the $z$-transform, $H_{0}(s)=\frac{1-e^{-T s}}{s}$ is the zero-order hold and $T$ is the sampling period. Moreover, we denote by $C_{d}(z)$ the transfer function of a discrete-time PID compensator which has the following structure:

$$
C_{d}(z)=K_{p}+K_{d} \frac{z-1}{z+1}+K_{i} \frac{z+1}{z-1},
$$

where the proportional and derivative gains $K_{p}, K_{d}$ and the integral gain $K_{i}$ are assumed to be real and positive. This transfer function has been obtained from the standard continuous time transfer function of a PID controller $C_{d}(s)=$ $\bar{K}_{p}+s \bar{K}_{d}+\frac{\bar{K}_{i}}{s}$ using the bilinear transformation with prewarping described by the substitutions

$$
s=\frac{\omega_{1}}{\tan \frac{\omega_{1} T}{2}}\left(\frac{z-1}{z+1}\right) \leftrightarrow \quad z=\frac{1+\frac{s}{\omega_{1}} \tan \frac{\omega_{1} T}{2}}{1-\frac{s}{\omega_{1}} \tan \frac{\omega_{1} T}{2}}
$$

where $\omega_{1}$ is the pre-warping frequency. The correspondence between the PID parameters in discrete and continuous time domains is the following:

$$
K_{d}=\bar{K}_{d} \frac{\omega_{1}}{\tan \frac{\omega_{1} T}{2}}, \quad K_{i}=\bar{K}_{i} \frac{\tan \frac{\omega_{1} T}{2}}{\omega_{1}}, \quad \bar{K}_{p}=K_{p}
$$

Notice that the gains $\bar{K}_{p}$ and $K_{p}$ are the same in the continuous and in the discrete time cases. The transfer function (1) can be rewritten as

$$
C_{d}(z)=K_{p}\left(1+T_{d} \frac{z-1}{z+1}+\frac{1}{T_{i}} \frac{z+1}{z-1}\right),
$$

where

$$
T_{i}=\frac{K_{p}}{K_{i}}, \quad T_{d}=\frac{K_{d}}{K_{p}}
$$

are respectively the integral and derivative time constants of the discrete PID controller. The design of the three parameters of the controller can be obtained considering two different cases related to the design specifications and the characteristic of the plant. In the first case the steadystate requirement leads to the imposition of the value of the integral term $K_{i}=K_{p} / T_{i}$. In this case the other two parameters can be used to meet specifications on the phase margin $\phi_{m}$ and the gain crossover frequency $\omega_{g}$. In the second case the pole $z_{p}=1$ of the PID controller is sufficient to guarantee that the steady-state requirement is satisfied. In this case two of the three parameters can be used to meet design requirements on the phase margin and the gain crossover frequency specifications. The third parameter can be used to satisfy other requirements. In this paper we consider design specifications on the ratio $T_{d} / T_{i}$, to guarantee that the zeros of the PID controller are real, and on the imposition of the gain margin $G_{m}$.

We now introduce some general concepts in order to solve the design problems considered in this paper using a graphical procedure. Let us start with some simple considerations on the Nyquist plane that will give us some insight into the graphical techniques presented in this paper. Loosely speaking, to meet specifications on the phase margin $\phi_{m}$ and the gain crossover frequency $\omega_{g}$, the loop gain frequency response $L(\omega, T)$ has to satisfy following relations

$$
\left|L\left(e^{j \omega_{g} T}\right)\right|=1, \quad \arg L\left(e^{j \omega_{g} T}\right)=\phi_{m}+\pi,
$$

and its Nyquist plot must exactly pass through the point $B$ of the unit circle with phase $\pi+\phi_{m}$ at frequency $\omega_{g} .{ }^{1}$ Intuitively, this means that the point $A$ of the frequency response of the discrete plant at frequency $\omega_{g}$, i.e., $A=H G\left(e^{j \omega_{g} T}\right)$, has to be brought to $B=e^{j\left(\pi+\phi_{m}\right)}$ by multiplication with the frequency response of the controller at frequency $\omega_{g}$. In other words, the equation $B=C_{d}\left(\omega_{g}, T\right) \cdot A$ must be satisfied. Stated differently, the control problem considered in this paper is to determine $C_{d}(\omega, T)$ such that $L\left(\omega_{g}, T\right)=e^{j\left(\pi+\phi_{m}\right)}$.

The set of all the points of the complex plane that can be brought to a desired point $B$ of the complex plane using a PID controller, the so called admissible domain for $B$, is not related to the structure of the plant, but only to the structure of the regulator and the position of point $B$.

\section{A. Imposition of the integral term $K_{i}>0$.}

Let us consider the case of given steady-state specifications that impose the value of the integral gain $K_{i}$. The other two degrees of freedom of the regulator can be imposed to meet specifications on the phase margin $\phi_{m}$ and the gain crossover frequency $\omega_{g}$.

First, the factor $\frac{K_{p}}{T_{i}} \frac{z+1}{z-1}$ can be separated from the transfer function

$$
\tilde{C}_{d}(z)=1+T_{i} \frac{z-1}{z+1}+T_{i} T_{d}\left(\frac{z-1}{z+1}\right)^{2},
$$

and viewed as part of the plant. In this way, the part of the controller to be designed is $\tilde{C}_{d}(z)$. Let

$$
\widetilde{H G}(z) \stackrel{\text { def }}{=} \frac{K_{p}}{T_{i}} \frac{z+1}{z-1} H G(z),
$$

and the loop gain transfer function can be written as $L(z)=$ $\tilde{C}_{d}(z) \widehat{H G}(z)$. The frequency response of (5) for $\omega \in[0, \pi / T]$ and sampling period $T$ is

$$
\tilde{C}_{d}\left(e^{j \omega T}\right)=M(\omega, T) e^{j \varphi(\omega, T)}=P(\omega, T)+j Q(\omega, T),
$$

where $M(\omega, T)$ and $\varphi(\omega, T)$ are the magnitude and the phase of $\tilde{C}_{d}\left(e^{j \omega T}\right)$, and

$$
P(\omega, T)=1-T_{d} T_{i} \tan ^{2} \frac{\omega T}{2}, \quad Q(\omega, T)=T_{i} \tan \frac{\omega T}{2} .
$$

We are ready to state the main result of this section.

Theorem 2.1: The equation $L\left(\omega_{g}, T\right)=e^{j\left(\pi+\phi_{m}\right)}$ holds with a PID controller (5) if and only if

$$
0<\varphi_{g}<\pi \quad \text { and } \quad M_{g} \cos \varphi_{g}<1
$$

\footnotetext{
${ }^{1}$ In most situations, this goal is enough to guarantee that the specifications on the phase (or gain) margins and crossover frequencies are met. However, that this can be only ensured when i) $L(z)=C_{d}(z) H G(z)$ has all poles in the unit circle and is strictly proper; and ii) the Nyquist plot of $L(\omega, T)$ for $\omega \geq 0$ intersects the unit circle and the negative real semi-axis only once. The second of these requirement is the one which guarantees that the stability margins are univocally determined.
} 
where

$$
\begin{gathered}
M_{g} \stackrel{\text { def }}{=} M\left(\omega_{g}\right)=1 /\left|\widetilde{H G}\left(e^{j \omega_{g} T}\right)\right|, \\
\varphi_{g} \stackrel{\text { def }}{=} \varphi\left(\omega_{g}\right)=\phi_{m}-\pi-\arg \widetilde{H G}\left(e^{j \omega_{g} T}\right) .
\end{gathered}
$$

If (7) are satisfied, the parameters of the PID controller $C_{d}(z)$ for which $L\left(\omega_{g}, T\right)=e^{j\left(\pi+\phi_{m}\right)}$ is satisfied are given by

$$
\begin{aligned}
T_{i} & =\frac{M_{g} \sin \varphi_{g}}{\tan \frac{\omega_{g} T}{2}}, \\
T_{d} & =\frac{1-M_{g} \cos \varphi_{g}}{M_{g} \sin \varphi_{g} \tan \frac{\omega_{g} T}{2}}, \\
K_{p} & =K_{i} \frac{M_{g} \sin \varphi_{g}}{\tan \frac{\omega_{g} T}{2}} .
\end{aligned}
$$

Proof: From (4), the controller (5) has to be designed in such a way that

$$
\tilde{C}_{d}\left(e^{j \omega_{g} T}\right)=M_{g} e^{j \varphi_{g}}
$$

holds, where $M_{g}$ and $\varphi_{g}$ satisfy (8) and (9). By equating real and imaginary parts of both sides of (6) and (11) we get

$$
\begin{gathered}
M_{g} \cos \varphi_{g}=1-T_{d} T_{i} \tan ^{2} \frac{\omega_{g} T}{2}, \\
M_{g} \sin \varphi_{g}=T_{i} \tan \frac{\omega_{g} T}{2} .
\end{gathered}
$$

Solving (12) and (13) with respect to $T_{i}$ and $T_{d}$ we find (10). The conditions (7) are obtained by imposing that the parameters of the PID controller are all positive.

The phase margin specification defines the position of the point $B$ that the loop gain frequency response has to cross, see Fig. 2. The admissible domain consisting of all the points $A$ that can be brought to $B$ by (5) can be defined as

$$
\mathscr{D}_{B}=\left\{A \in \mathbb{C} \mid \exists T_{i}, T_{d}>0, \exists \omega, T \geq 0: \tilde{C}_{d}\left(e^{j \omega T}\right) \cdot A=B\right\} .
$$

This domain is represented by the shaded area shown in Fig. 2. This domain corresponds to the set of all the points $A=M_{A} e^{j \varphi_{A}}$ that can be brought to point $B=e^{j \varphi_{B}}$ such that $\varphi_{g}=\varphi_{B}-\varphi_{A}$ verifies (7).

The problem admits a solution if and only if $A=$ $\widetilde{H G}\left(e^{j \omega_{g} T}\right)$ belongs to the considered admissible domain $\mathscr{D}_{B}$.

Example 2.1: Let us consider the discrete-time plant $H G(z)$ obtained from the discretisation of the transfer function

$$
G(s)=\frac{14(s+1)}{s(s+1.5)^{2}(s+3)},
$$

using a zero-order hold with a sampling period $T=0.05 \mathrm{sec}$. Design a PID compensator that meets the following specifications: acceleration constant $K_{a}=2$; phase margin $\phi_{m}=50^{\circ}$; gain crossover frequency $\omega_{g}=1.6 \mathrm{rad} / \mathrm{s}$.

Solution: The steady-state requirement is satisfied imposing the value of the integral constant $K_{i}=0.024$. The desired magnitude and the phase of the controller (5) at frequency $\omega_{g}$ are $M_{g}=1.64$ and $\varphi_{g}=2.026 \mathrm{rad}$, respectively. These values

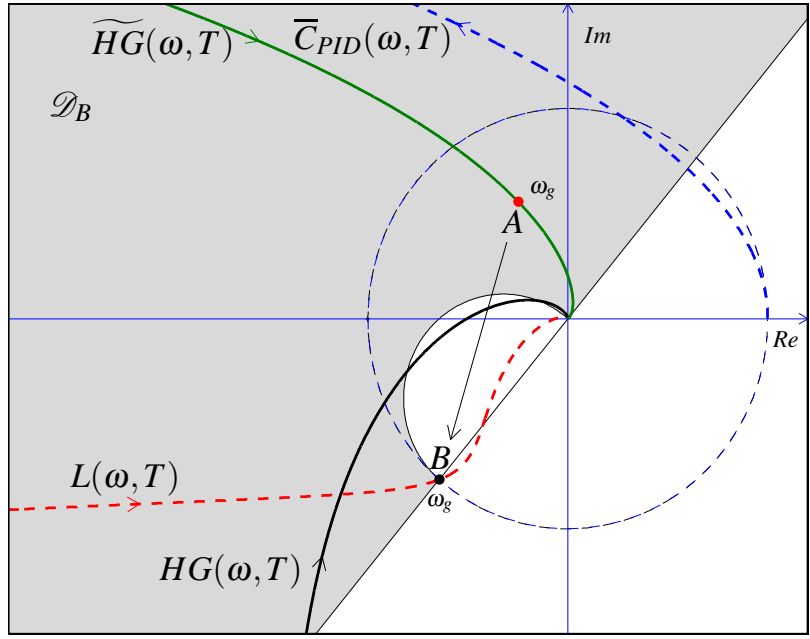

Fig. 2. Graphical solution of Example 2.1 on the Nyquist plane.

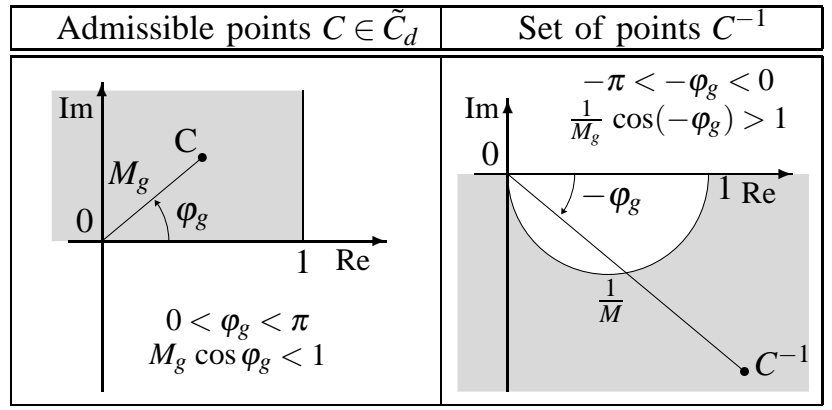

Fig. 3. Graphical representation of admissible of points $C$ of $\tilde{C}_{d}\left(e^{j \omega T}\right)$ and their inverse points $C^{-1}=\frac{1}{C}$.

satisfy (7). Notice that (7) can be graphically verified drawing the admissible domain of (5) with respect to point $B=e^{j 230^{\circ}}$ and verifying that point $A=\widetilde{H G}\left(e^{j \omega_{g} T}\right)$ belongs to this domain. Indeed, we see from Fig. 2 that in this example point $A$ belongs to the shaded region representing the admissible domain. As a consequence, the problem admits solution with an admissible PID controller as expected by virtue of Theorem 2.1. Indeed, using (10) we compute the time constants of the PID controller, and we obtain the values $T_{i}=36.9 \mathrm{sec}$ and $T_{d}=29.14 \mathrm{sec}$ which are indeed positive. Thus, the closed loop system is asymptotically stable, and the constraints on the steady-state performance, on the phase margin and on the gain crossover frequency are satisfied.

\section{B. Imposition of the ratio $T_{d} / T_{i}$}

Let us consider the case in which the steady-state specifications do not constrain the value of the integral term $K_{i}$. In this case the three parameters of the controller can be designed to meet specifications on the phase margin $\phi_{m}$, the gain crossover frequency $\omega_{g}$ and a third parameter. We first consider the case in which the ratio $T_{d} / T_{i}$ is assigned. Values of $\sigma^{-1}=T_{i} / T_{d} \geq 4$ guarantee that the zeros of the discretetime controller $\tilde{C}_{d}(z)=\frac{N(z)}{D(z)}$ are real. The following theorem is the discrete counterpart of a result in [6, p. 140]. The frequency response of (1) for $\omega \in[0, \pi / T]$ and sampling 
period $T$ is

$$
C_{d}\left(e^{j \omega T}\right)=M(\omega, T) e^{j \varphi(\omega, T)}=P+j Q(\omega, T),
$$

where

$$
P=K_{p}, \quad Q(\omega, T)=K_{p} T_{d} \tan \frac{\omega T}{2}-\frac{1}{T_{i}} \frac{K_{p}}{\tan \frac{\omega T}{2}} .
$$

Theorem 2.2: Referring to the system shown in Fig. 1, the equation $L\left(\omega_{g}, T\right)=e^{j\left(\pi+\phi_{m}\right)}$ holds with an admissible PID controller (1) - or (2) - if and only if

$$
\varphi_{g} \in(-\pi / 2, \pi / 2)
$$

where

$$
\varphi_{g} \stackrel{\text { def }}{=} \varphi\left(\omega_{g}\right)=\phi_{m}-\pi-\arg H G\left(e^{j \omega_{g} T}\right) .
$$

If (16) is satisfied, the parameters of the PID controller $C_{d}(z)$ for which $L\left(\omega_{g}, T\right)=e^{j\left(\pi+\phi_{m}\right)}$ is satisfied are given by

$$
\begin{aligned}
K_{p} & =M_{g} \cos \varphi_{g}, \\
T_{i} & =\frac{\tan \varphi_{g}+\sqrt{\tan ^{2} \varphi_{g}+4 \sigma}}{2 \sigma \tan \frac{\omega_{g} T}{2}}, \\
T_{d} & =T_{i} \sigma .
\end{aligned}
$$

where

$$
M_{g} \stackrel{\text { def }}{=} M\left(\omega_{g}\right) \frac{1}{\left|H G\left(e^{j \omega_{g} T}\right)\right|} .
$$

Proof: From (4), the controller (2) has to be designed in such a way that

$$
C_{d}\left(e^{j \omega_{g} T}\right)=M_{g} e^{j \varphi_{g}}
$$

holds, where $\varphi_{g}$ satisfy (16). By equating real and imaginary parts of both sides of (15) and (20) we get

$$
\begin{gathered}
M_{g} \cos \varphi_{g}=K_{p}, \\
M_{g} \sin \varphi_{g}=K_{p} T_{d} \Omega\left(\omega_{p}\right)-\frac{1}{T_{i}} \frac{K_{p}}{\Omega\left(\omega_{p}\right)} .
\end{gathered}
$$

Solving (21) and (22) with respect to $T_{i}$ and $T_{d}$ we find (18). The condition (16) is obtained by imposing that the parameters of the PID controller are all positive.

Example 2.2: Let us consider the discrete-time plant $H G(z)$ obtained from the discretisation of the continuoustime plant described by the transfer function

$$
G(s)=\frac{6(s+1)}{s(s+1.5)^{2}(s+3)} e^{-0.8 s},
$$

using a zero-order hold with a sampling period $T=0.04 \mathrm{sec}$. The task is to design the discrete-time PID regulator (2) that meets the following specifications: zero velocity error; phase margin $\phi_{m}=50^{\circ}$; gain crossover frequency $\omega_{g}=1.3 \mathrm{rad} / \mathrm{sec}$.

Solution. The steady-state specification is automatically satisfied by the use alone of a discrete time PID controller. The extra freedom is used to obtain $\sigma=1 / 8$ (so that $\sigma^{-1}>$ 4). Then the desired magnitude and phase of the controller at frequency $\omega_{g}$ are $M_{g}=1.70$ and $\varphi_{g}=1.147 \mathrm{rad}$, respectively. Since $\varphi_{g} \in(-\pi / 2, \pi / 2)$, the condition of Theorem 2.2 is

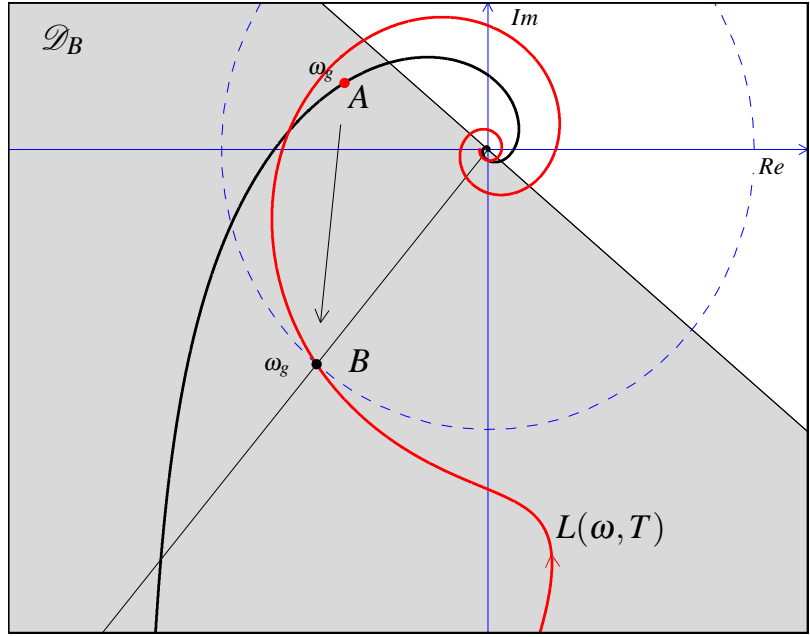

Fig. 4. Graphical solution of Example 2.2 on the Nyquist plane.

satisfied, and therefore the problem admits a solution. Using (18) we find $K_{p}=0.472, T_{i}=1.08 \cdot 10^{3} \mathrm{sec}$ and $T_{d}=134.6$ sec. The loop frequency response is shown in red in Fig. 4.

\section{Imposition of the gain margin}

In this case the third parameter of the controller is designed to assign the gain margin to a certain value $G_{m}$. This leads to a loop gain frequency response that passes through the point $\left(-1 / G_{m}, 0\right)$ at the phase crossover frequency $\omega_{p}$, i.e.,

$$
\left|L\left(e^{j \omega_{p} T}\right)\right|=\frac{1}{G_{m}} \quad \arg L\left(e^{j \omega_{p} T}\right)=-\pi .
$$

Therefore, the three parameters of the controller must be calculated to simultaneously verify (4) and (24).

The frequency response of (2) at frequency $\omega_{p}$ can be expressed as

$$
C_{d}\left(e^{j \omega_{p} T}\right)=K_{p}+j\left(K_{p} T_{d} \tan \frac{\omega_{p} T}{2}-\frac{1}{T_{i}} \frac{K_{p}}{\tan \frac{\omega_{p} T}{2}}\right),
$$

or it can be written in polar form as

$$
C_{d}\left(e^{j \omega_{p} T}\right)=M_{p} e^{j \varphi_{p}}=M_{p} \cos \left(\varphi_{p}\right)+j M_{p} \sin \left(\varphi_{p}\right) .
$$

By equating real and imaginary part of (25) and (26) we obtain

$$
\begin{gathered}
M_{p} \cos \varphi_{p}=K_{p}, \\
M_{p} \sin \varphi_{p}=K_{p} T_{d} \Omega\left(\omega_{p}\right)-\frac{1}{T_{i}} \frac{K_{p}}{\tan \frac{\omega_{p} T}{2}} .
\end{gathered}
$$

These equations must be satisfied together with (21) and (22). Thus, the value of $\omega_{p}$ can be obtained as solution of the following equation

$$
M_{g} \cos \varphi_{g}=M_{p} \cos \varphi_{p} .
$$

which follows directly by (21) and (27). By following the same argument used in the proof of Lemma 1 in [15], 
it is easy to see that when the transfer function of the plant is rational in $z,(29)$ is a polynomial equation in $\omega_{p}$, and therefore its solutions can be calculated with arbitrary precision.

Theorem 2.3: The parameters of PID controller $C_{d}(z)$ for which $L\left(\omega_{g}, T\right)=e^{j\left(\pi+\phi_{m}\right)}$ and $L\left(\omega_{p}, T\right)=\frac{1}{G_{m}} e^{-j \pi}$ are simultaneously satisfied are

$$
\begin{aligned}
K_{p} & =M_{g} \cos \varphi_{g}, \\
T_{i} & =\frac{\Omega_{g}^{2}-\Omega_{p}^{2}}{\Omega_{g} \Omega_{p}\left(\Omega_{p} \tan \varphi_{g}-\Omega_{g} \tan \varphi_{p}\right)}, \\
T_{d} & =\frac{\Omega_{g} \tan \varphi_{g}-\Omega_{p} \tan \varphi_{p}}{\Omega_{g}^{2}-\Omega_{p}^{2}},
\end{aligned}
$$

where $\varphi_{g}=\phi_{m}+\pi-\arg H G\left(e^{j \omega_{g} T}\right), M_{g}=1 /\left|H G\left(e^{j \omega_{g} T}\right)\right|$, $\Omega_{g}=\tan \frac{\omega_{g} T}{2}, \Omega_{p}=\tan \frac{\omega_{p} T}{2}$ and $\omega_{p}$ is the solution of (29), with $M_{p}=1 /\left(G_{m}\left|H G\left(e^{j \omega_{p} T}\right)\right|\right)$ and $\varphi_{p}=\pi-\arg H G\left(e^{j \omega_{p} T}\right)$. The solution leads to an admissible PID controller (i.e., one in which the three parameters are all non-negative) if and only if $\varphi_{g} \in(-\pi / 2, \pi / 2)$ and $\omega_{p}$ verifies the following constraints

$$
\left\{\begin{array} { l } 
{ \omega _ { p } < \omega _ { g } } \\
{ \Omega _ { g } \operatorname { t a n } \varphi _ { g } > \Omega _ { p } \operatorname { t a n } \varphi _ { p } } \\
{ \Omega _ { p } \operatorname { t a n } \varphi _ { g } > \Omega _ { g } \operatorname { t a n } \varphi _ { p } }
\end{array} \text { or } \quad \left\{\begin{array}{l}
\omega_{p}>\omega_{g} \\
\Omega_{g} \tan \varphi_{g}<\Omega_{p} \tan \varphi_{p} \\
\Omega_{p} \tan \varphi_{g}<\Omega_{g} \tan \varphi_{p}
\end{array}\right.\right.
$$

Proof: The problem is solvable if and only if $\omega_{p}$ is a solution of (29). From (21) and (22), and from (27) and (28) we get

$$
\begin{aligned}
-\Omega_{g} T_{i} \tan \varphi_{g} & =1-\Omega_{g}^{2} T_{i} T_{d}, \\
-\Omega_{p} T_{i} \tan \varphi_{p} & =1-\Omega_{p}^{2} T_{i} T_{d} .
\end{aligned}
$$

Solving (34) and (35) in $T_{i}$ and $T_{d}$ leads to (31) and (32), and $K_{p}$ is positive only if $\varphi_{g} \in(-\pi / 2, \pi / 2)$. Moreover, the constraints $T_{i}>0$ and $T_{d}>0$ are verified if (33) are satisfied.

The Nyquist plot of $C_{d}\left(e^{j \omega T}, K_{p}\right)$, which shapes the loop gain frequency response $L(\omega, T)$ to pass through the point $B_{g}=e^{j\left(\pi+\phi_{m}\right)}$ at the gain crossover frequency $\omega_{g}$, is the straight line with modulus $K_{p}$ given by (30), see [28]. The loop gain frequency response $L(\omega, T)$ also passes through point $B_{p}=\frac{1}{G_{m}} e^{j \pi}$ at frequency $\omega_{p}$ (so that $L\left(\omega_{p}, T\right)=$ $\left.C_{d}\left(e^{j \omega_{p} T}, K_{p}\right) H G\left(\omega_{p}, T\right)=B_{p}\right)$ if and only if

$$
H G\left(\omega_{p}, T\right)=B_{p} / C_{d}\left(e^{j \omega_{p} T}, K_{p}\right) .
$$

Since the plot of $\frac{1}{C_{d}\left(e^{j \omega T}, K_{p}\right)}$ is a circle having as diameter the segment $\left(0,1 / K_{p}\right)$, see [28], the plot of $B_{p} / C_{d}\left(e^{j \omega_{p} T,}, K_{p}\right)$ is the circle with diameter $\left(0, B_{p} / K_{p}\right)$. It follows that solutions of (29) are the frequencies $\omega_{p}$ of $H G(\omega, T)$ at intersection points with the circle having as diameter the segment $\left(0, B_{p} / K_{p}\right)$.

Example 2.3: Let us consider the discrete-time plant $H G(z)$ obtained using the non-minimum phase plant

$$
G(s)=\frac{s^{3}-3.7 s^{2}+s+2.5}{s^{5}+6 s^{4}+40 s^{3}+43 s^{2}+43 s+17} e^{-1.2 s},
$$

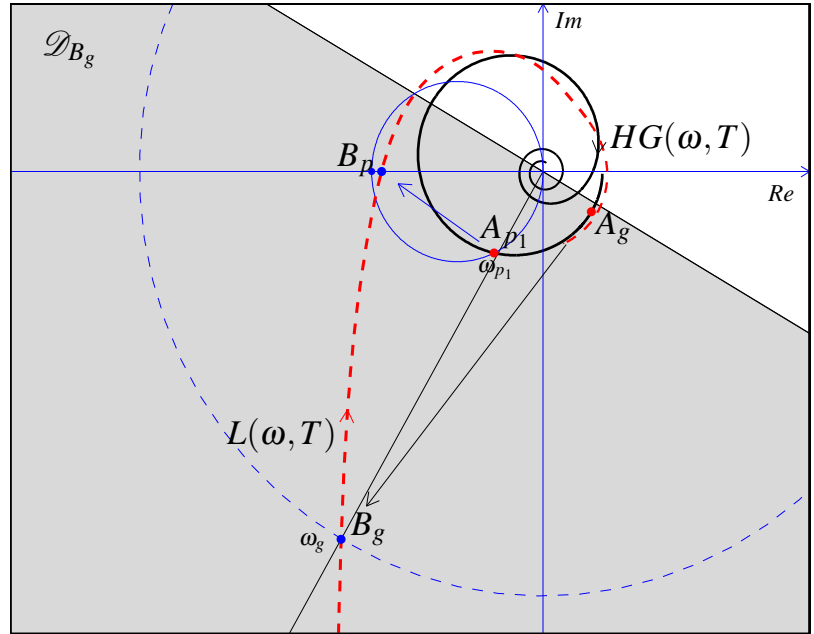

Fig. 5. Graphical solution of Example 2.3 realted to design specifications on the phase and gain margins, and the gain crossover frequency.

and a zero-order hold with a sampling period $T=0.04 \mathrm{sec}$. Design a discrete-time PID compensator $C_{d}(z)$ to meet the following design specifications: phase margin $\phi_{m}=60^{\circ}$, gain margin $G_{m}=2.5$ and gain crossover frequency $\omega_{g}=0.2$ $\mathrm{rad} / \mathrm{sec}$.

Solution: The values of the desired magnitude and phase of the controller at frequency $\omega_{g}$ are $M_{g}=6.55$ and $\varphi_{g}=$ $-1.43 \in(-\pi / 2, \pi / 2)$, respectively. Notice that point $A_{g}=$ $H G\left(e^{j \omega_{g} T}\right)$ belongs to the domain $\mathscr{D}_{B_{g}}$, see Fig. 5. From (30) it follows that $K_{p}=0.94$. The solutions of (29) are $\omega_{p i} \in$ $\{0.62,0.95,2.41,3.97 \ldots\}$ and correspond to the frequencies at the intersections points of $H G(\omega, T)$ with the circle whose diameter coincides with the segment $\left(0, B_{p} / K_{p}\right)$, see Fig. 6. One possible solution is obtained by bringing the point $A_{p_{1}}$ at frequency $\omega_{p 1}$ to point $B_{p}$. From (31) and (32) the other designed parameters are $T_{i}=35.14 \mathrm{sec}$ and $T_{d}=$ $59.07 \mathrm{sec}$. The corresponding loop gain frequency response $L(\omega, T)$ is represented by the dashed red line in Fig. 5.

\section{REFERENCES}

[1] J.L. Meza, V. Santibanez, R. Soto, M.A. Llama, Fuzzy Self-Tuning PID Semiglobal Regulator for Robot Manipulators. IEEE Transactions on Industrial Electronics, vol. 59, no. 6, pp. 2709-2717, June 2012.

[2] B. Lennartson, B. Kristiansson, Evaluation and tuning of robust PID controllers. IET Control Theory \& Applications, vol.3, no.3, pp. 294302, March 2009.

[3] P.H. Chang, J.H. Jung, A Systematic Method for Gain Selection of Robust PID Control for Nonlinear Plants of Second-Order Controller Canonical Form. IEEE Transactions on Control Systems Technology, vol. 17, no. 2, pp. 473-483, March 2009.

[4] J.G. Ziegler, and N.B. Nichols, Optimum settings for automatic controllers. Trans. ASME, vol. 64, pp. 759-768, 1942.

[5] K.J. Aström, and T. Hagglund, PID Controllers: Theory, Design, and Tuning, second edition. Instrument Society of America, Research Triangle Park, NC, 1995.

[6] K.J. Aström, and T. Hagglund, Advanced PID Control. Instrument Society of America, Research Triangle Park, NC, 2006.

[7] A. Datta, M.-T. Ho, and S.P. Bhattacharyya. Structure and Synthesis of PID Controllers. Springer, 2000.

[8] A. Visioli, Practical PID Control. Advances in Industrial Control, Springer-Verlag, 2006. 


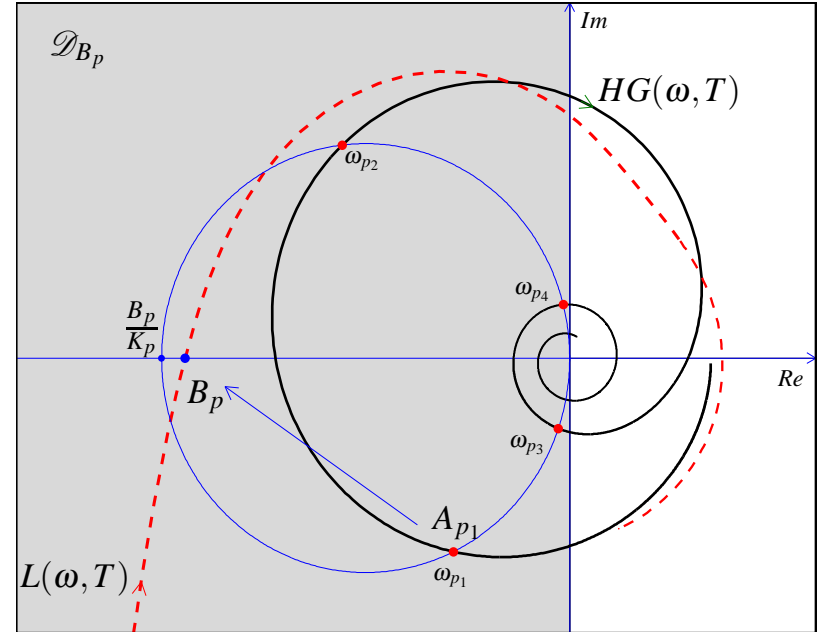

Fig. 6. Example 2.3: graphical solution of eq. (29) to determine $\omega_{p i}=$ $\left(\omega_{p 1}, \omega_{p 2}, \omega_{p 3}, \omega_{p 4}, \ldots\right)$

[9] K.S. Yeung, and K.H. Lee. A Universal Design Chart for Linear TimeInvariant Continuous-Time and Discrete-Time Compensators. IEEE Transactions on Education, E-43, no. 3, pp. 309-315, 2000.

[10] S. Skogestad. Simple analytic rules for model reduction and PID controller tuning. Journal of Process Control, vol. 13, pp. 291-309, 2003.

[11] K. Kim, and Y.C. Kim,, The Complete Set of PID Controllers with Guaranteed Gain and Phase Margins. Proceedings of the $44^{\text {th }}$ IEEE Conference on Decision and Control, and the European Control Conference 2005, pp. 6533-6538, Seville, Spain, December 12-15, 2005.

[12] L.H. Keel and S.P. Bhattacharyya. Controller Synthesis Free of Analytical Models: Three Term Controllers. IEEE Transactions on Automatic Control, AC-53, no. 6, pp. 1353-1369, 2008.

[13] W.K. Ho, and C.C. Hang, and L.S. Cao, Tuning of PID Controllers Based on Gain and Phase Margin Specifications. Automatica, vol. 31, no. 3, pp. 497-502, 1995.

[14] C.L. Phillips, and H.T. Nagle. Digital Control Systems. Prentice Hall, 1995.

[15] L. Ntogramatzidis, and A. Ferrante, Exact Tuning of PID Controllers in Control Feedback Design, IET Control Theory \& Applications, vol. 5, no. 4, pp. 565-578, 2011.

[16] G. Marro and R. Zanasi, New Formulae and Graphics for Compensator Design, IEEE International Conference On Control Applications, Trieste, Italy, September 1-4, 1998.

[17] R. Zanasi, S. Cuoghi and L. Ntogramatzidis, Analytical and Graphical Design of Lead-Lag Compensators. International Journal of Control, vol. 84, no. 11, pp. 1830-1846, 2011.

[18] R. Zanasi, S. Cuoghi, and L. Ntogramatzidis, "Analytical Design of Lead-Lag Compensators on Nyquist and Nichols planes". In Proceedings of the $18^{\text {th }}$ International Federation of Automatic Control (IFAC) World Congress (IFAC) World Congress, Milan, Italy, Aug. 28 - Sept. 2, 2011.

[19] R. Zanasi, S. Cuoghi, and L. Ntogramatzidis, "Lead-Lag compensators: analytical and graphical design on the Nyquist plane". In Proceedings of the $50^{\text {th }}$ IEEE Conference on Decision and Control (CDC 11), Orlando, FL, USA, Dec. 12-15, 2011.

[20] H. Zhang, Y. Shi and A.S. Mehr, Robust $\mathscr{H}_{\infty}$ PID control for multivariable networked control systems with disturbance/noise attenuation. International Journal of Robust and Nonlinear Control, vol. 22, no. 2, pp. 183-204, 2012.

[21] H. Zhang, Y. Shi and A.S. Mehr, Robust static output feedback control and remote PID design for networked motor systems. IEEE Transactions on Industrial Electronics, vol. 58, no. 12, pp. 5396-5405, 2011.

[22] N.S. Nise, Control Systems Engineering, 5rd Edition, Wiley, Hoboken, NJ, 2008.

[23] K. Ogata, Modern Control Engineering, 4th Edition, Prentice Hall, Upper Saddle River, NJ, 2009.
[24] R.C. Dorf and R.H. Bishop, Modern Control Systems, Prentice Hall, Upper Saddle River, NJ, 2008.

[25] K. Ogata, Discrete-Time Control Systems, 2nd edn. Prentice Hall, Upper Saddle River, NJ, 1995.

[26] W.R. Wakeland. Bode Compensation Design. IEEE Transactions on Automatic Control, AC-21, no. 5, pp. 771-773, 1976.

[27] R. Zanasi and S. Cuoghi, Analytical and Graphical Design of PID Compensators on the Nyquist plane. IEEE IFAC Conference on Advances in PID Control PID'12, International Federation of Automatic Control, Brescia, Italy, 28-30 March 2012.

[28] R. Zanasi and S. Cuoghi, Direct methods for the synthesis of PID compensators: analytical and graphical design. IEEE IECON, Industrial Electronics Conference, 7-10/11/2011, Melbourne, Australia. 